\title{
10 K Room Temperature LNA for SKA Band 1
}

\author{
J. Schleeh ${ }^{1}$, N. Wadefalk ${ }^{1}$, P. Å. Nilsson ${ }^{2}$, J. Grahn ${ }^{2}$ \\ ${ }^{1}$ Low Noise Factory, Gothenburg, SE-412 63, Sweden \\ ${ }^{2}$ GigaHertz Centre, Department of Microtechnology and Nanoscience, \\ Chalmers University of Technology, Gothenburg, SE-412 96, Sweden
}

\begin{abstract}
A room temperature LNA suitable for Square Kilometer Array band $1(0.35-1.05 \mathrm{GHz})$ has been designed, fabricated and tested. The design is based on InP HEMTs, and focused on minimizing losses in the input matching network. Noise measurement methods in two different labs were used to confirm the $10 \mathrm{~K}$ noise temperature of the LNA. The gain was flat at $50 \mathrm{~dB}$ and the input and output return loss better than $10 \mathrm{~dB}$ in most of the band.

Index Terms - InP HEMT, Low Noise Amplifier, SKA.
\end{abstract}

\section{INTRODUCTION}

The Square Kilometer Array (SKA) is the next-generation radio telescope, combining several thousands of antennas into a total surface area approaching one square kilometer. The array is divided in three types of receivers, the low-frequency aperture array, the mid-frequency aperture array, and the dishes. In the first phase, the dishes (15 m offset Gregorian) are planned to cover 0.35 to $13.8 \mathrm{GHz}$, in 5 separate frequency bands. [1]

To minimize system noise temperature, receivers and antenna feeds should be cooled to cryogenic temperatures. For the band 1 feed $(0.35-1.05 \mathrm{GHz})$, however, cooling is not cost effective as a vacuum window of more than $1 \mathrm{~m}$ of diameter would be needed. The low noise amplifier (LNA) can still be cooled, but since room temperature connectors, vacuum feedthrough and a cable are needed to connect the cryogenic LNA with the feed, a substantial amounts of noise is added. Even with as little as $1 \mathrm{~K}$ LNA noise, the total receiver noise will likely end up close to $10 \mathrm{~K}$. If the same, or better, noise performance could be obtained with a room temperature LNA, not only a much simpler receiver could be used, but also a lot of electrical power would be saved. Each cryogenic receiver consumes a few kilowatts. In a fully operating SKA, this translates into several Megawatts of power.

In this paper, we present a room temperature LNA for SKA band 1. The LNA is designed to be connected directly to the feed, with the feeding pin soldered directly to the input matching network to minimize losses (SMA connector is removed compared to Fig. 1). Measured at room temperature with an SMA input connector, the LNA achieves a flat gain of $50 \mathrm{~dB}$ and a lowest noise temperature of $10 \mathrm{~K}$. This is directly comparable to the feed interface of a cryogenic receiver, but without any cooling required.

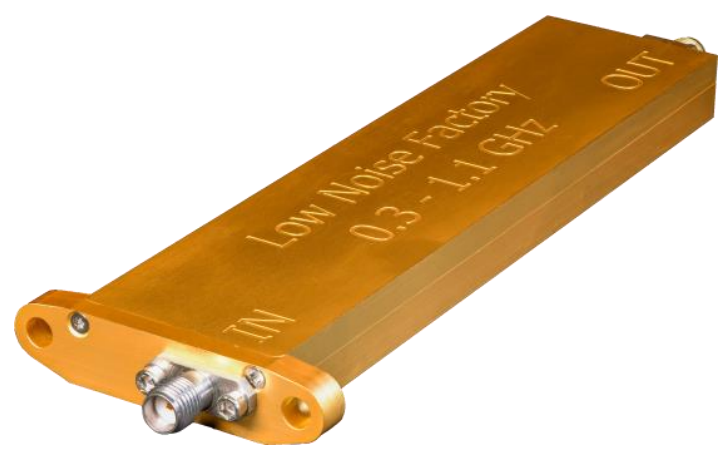

Fig. 1. Input side of the $127 \times 47 \times 10.5 \mathrm{~mm}$ LNA chassis (excluding connectors). The input connector is replaced with the feed pin when installed in the antenna.

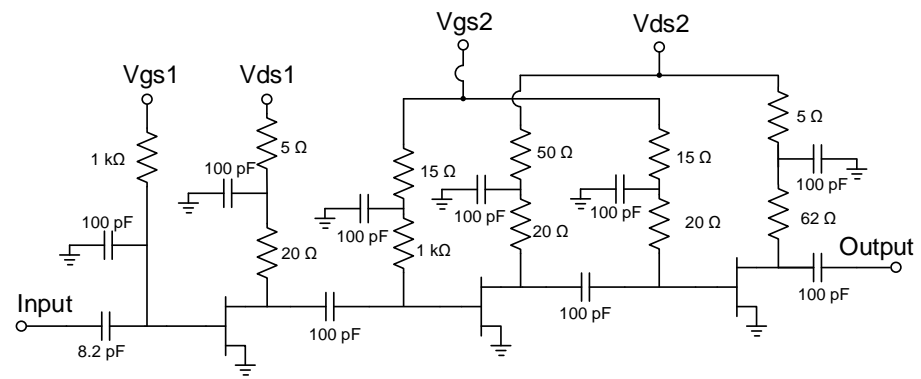

Fig. 2. Schematic of the 3 -stage LNA

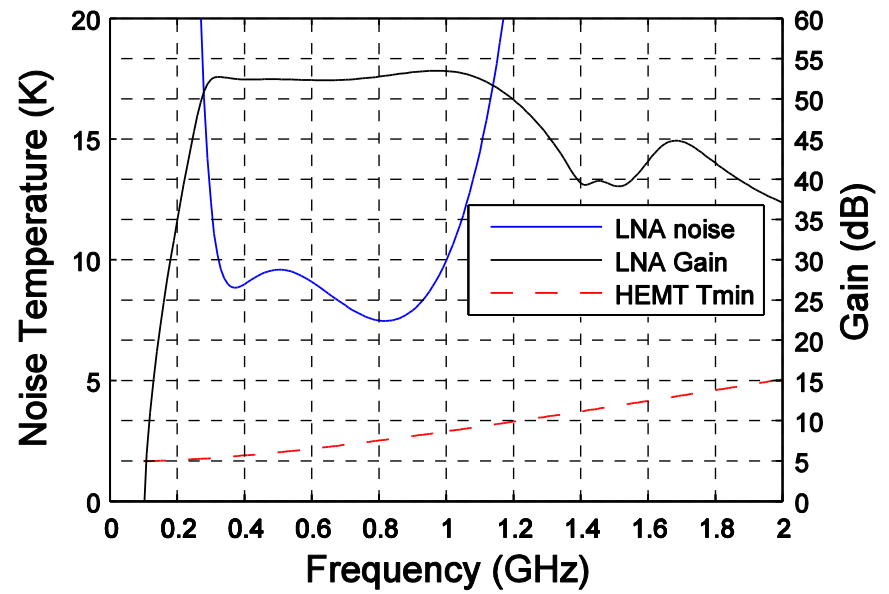

Fig. 3. Simulated noise and gain of the LNA together with the minimum noise temperature of the transistor. 
TABLE 1

SMALL-SIGNAL MODEL VALUES OF THE 4X50 $\mu \mathrm{m}$ (EXTRACTED) AND 12X50 $\mu \mathrm{m}$ (SCALED) INP HEMT AT OPTIMUM LOW NOISE BIAS. UNITS ARE $\mathrm{V}, \mathrm{mA}, \mathrm{mS}, \Omega, \mathrm{fF}, \mathrm{pH}$ AND $\mathrm{K}$.

\begin{tabular}{ccc}
\hline \hline & $4 \times 50 \mu \mathrm{m}$ & $12 \times 50 \mu \mathrm{m}$ \\
\hline$C_{\mathrm{gs}}$ & 110 & 330 \\
$C_{\mathrm{gd}}$ & 29 & 88 \\
$C_{\mathrm{ds}}$ & 54 & 162 \\
$g_{\mathrm{m}}$ & 204 & 611 \\
$R_{\mathrm{i}}$ & 3.3 & 1.1 \\
$R_{\mathrm{j}}$ & 9.6 & 3.2 \\
$G_{\mathrm{ds}}$ & 13 & 40 \\
$R_{\mathrm{g}}$ & 0.5 & 0.17 \\
$R_{\mathrm{d}}$ & 3.3 & 1.1 \\
$R_{\mathrm{s}}$ & 1.3 & 0.4 \\
$T_{\mathrm{d}}$ & 4000 & 4000 \\
\hline \hline
\end{tabular}

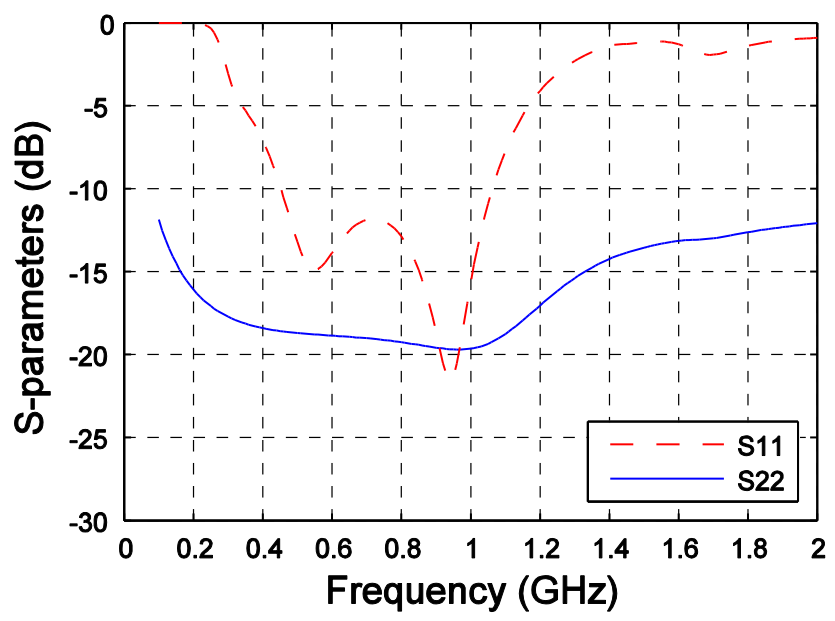

Fig. 4. Simulated S11 and S22 of the LNA.

\section{DESIGN}

To compete with cryogenic amplifiers at room temperature, a really good low noise transistor and a close to lossless input matching network is required. One transistor with a good track record both at room temperature and cryogenic is the InP high electron mobility transistor (HEMT) [2, 3]. The minimum noise temperature $\left(T_{\min }\right)$ of the $130 \mathrm{~nm}$ bare die transistors obtained for this work is less than $3 \mathrm{~K}$ at $1 \mathrm{GHz}$, see Fig. 3. (Based on small signal noise modelling of $4 \times 50 \mu \mathrm{m}$ InP HEMTs as described in $[4,5])$. All intrinsic small signal noise parameters of the model are given in Table 1.

To get a good input match of the LNA at 0.35 to $1.05 \mathrm{GHz}$, and allow for noise matching, a larger transistor than 4x50 $\mu \mathrm{m}$ is needed. These larger transistors were not stable when probed at $50 \Omega$, however. A less elegant, but satisfactory, solution was to scale the $4 \times 50 \mu \mathrm{m}$ model linearly with number of fingers and gate width to a more suitable size for the frequency of the LNA. The parameters of the $12 \times 50 \mu \mathrm{m}$ transistor (scaled from $4 \times 50 \mu \mathrm{m}$ ), which was finally chosen for the first stage, are shown in Table 1. For stage 2 and 3, less capacitance is needed, and the $4 \times 50 \mu \mathrm{m}$ size was chosen. All

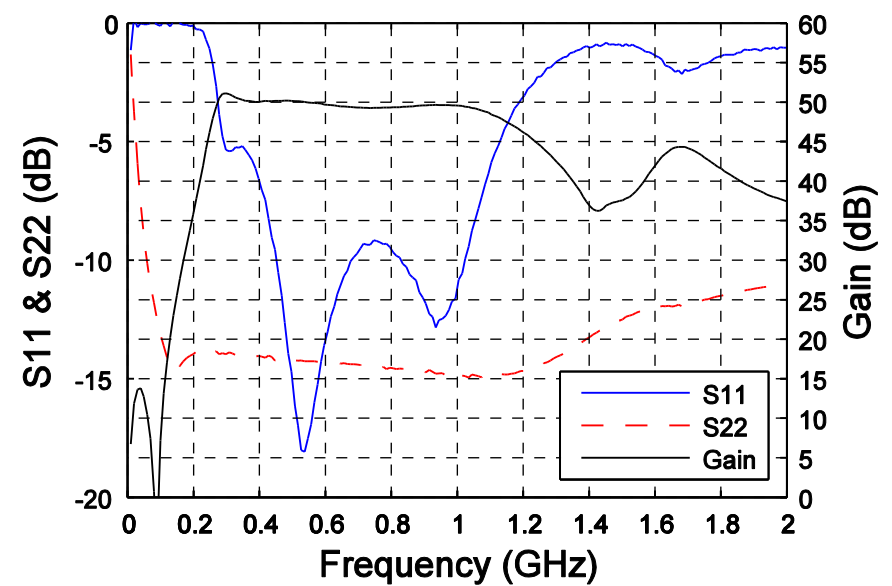

Fig. 5. Measured S-parameters of the LNA. Bias: stage $1, V_{\mathrm{d}}=1.5 \mathrm{~V}$, $I_{\mathrm{d}}=45 \mathrm{~mA}$, stage $2+3, V_{\mathrm{d}}=1.8 \mathrm{~V}, I_{\mathrm{d}}=35 \mathrm{~mA}$

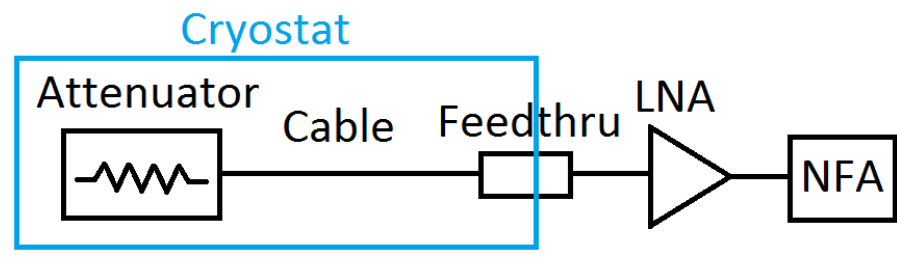

Fig. 6. Noise measurement setup.

stages were matched using a combination of reactive and resistive elements together with the shorted stubs used for biasing the transistors. The schematic of the LNA is shown in Fig. 2.

In low frequency LNAs where the transistor noise is very low, the metal and dielectric loss of the input matching network can contribute significantly to the overall LNA noise. $0.01 \mathrm{~dB}$ loss translates to about $0.7 \mathrm{~K}$ extra noise temperature of the LNA. The solution is a coaxial airline design where a pin is suspended inside the ground metal chassis with only air as dielectric, or a suspended microstrip line with a very thin substrate. Theoretically the coaxial version is slightly better than the suspended microstrip, but we couldn't find a good solution for providing gate bias for the first stage, which was very easily done with a suspended microstrip. For this reason, and for a much easier fabrication process, the suspended microstrip line solution was chosen. The substrate of the suspended microstrip was 5 mil Rogers 6002 suspended 4 mm over and under the ground chassis.

Simulated noise and S-parameters of the LNA are shown in Fig. 3 and Fig. 4.

\section{MEASUREMENTS}

S-parameters, measured using an Agilent 67 GHz PNA, can be seen in Fig. 5. All three curves have the same shape as simulations, but S11 and S22 are slightly worse in magnitude.

To accurately measure noise temperature is much more complicated. Specified ENR uncertainty of the popular 


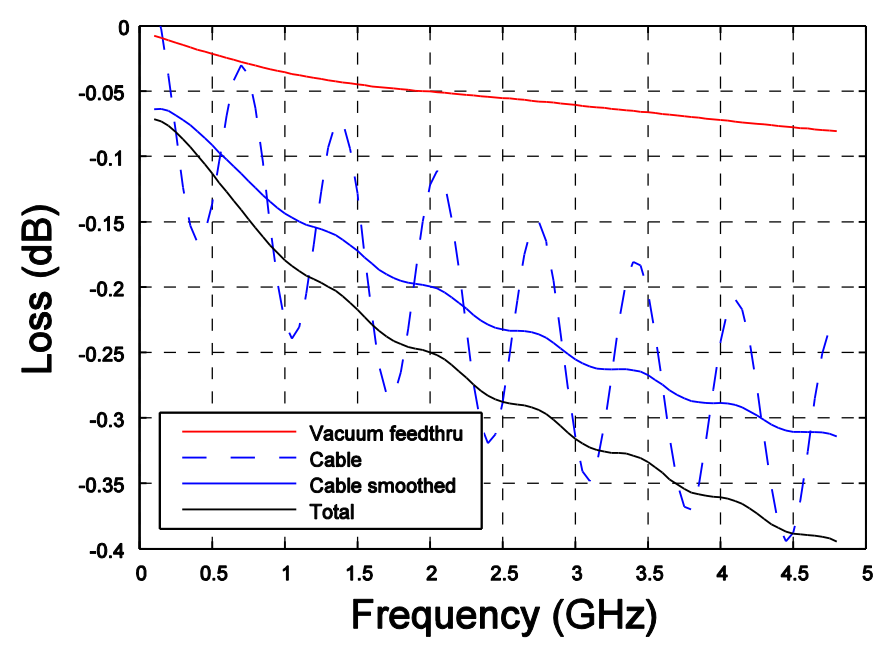

Fig. 7. Losses of the vacuum feedthrough and cryogenic cable measured with a VNA.

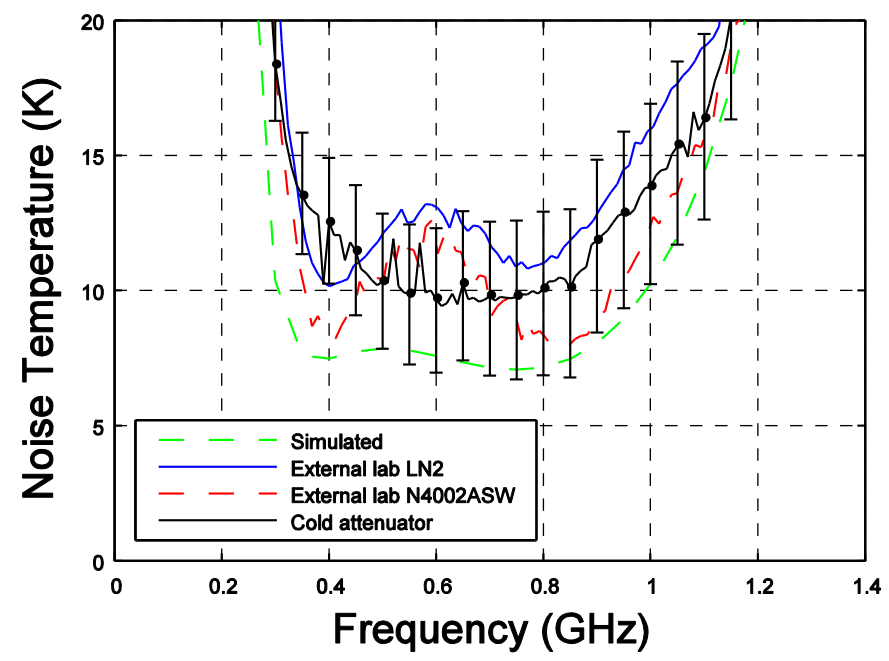

Fig. 8. Measured noise temperature of the LNA. Bias: stage $1, V_{\mathrm{d}}=$ $1.5 \mathrm{~V}, I_{\mathrm{d}}=45 \mathrm{~mA}$, stage $2+3, V_{\mathrm{d}}=1.8 \mathrm{~V}, I_{\mathrm{d}}=35 \mathrm{~mA}$

Keysight N4002 noise source (14 dB ENR) is $0.15 \mathrm{~dB}$ [6]. For a $10 \mathrm{~K}$ LNA, only this ENR uncertainty corresponds to a noise uncertainty interval between -1.2 and $21.7 \mathrm{~K}$, i.e. more than $100 \%$ of the noise temperature. Reducing the ENR by using the $6 \mathrm{~dB}$ N4000A noise source makes things even worse. With this noise source the uncertainty lies between -11.7 and $34.4 \mathrm{~K}$.

A better approach is to reduce the hot and cold load temperatures to be of the same magnitude as the LNA noise temperature. One way to achieve this is to use a $20 \mathrm{~dB}$ cold attenuator in a calibrated cryogenic noise measurement setup. By attaching a heater to the attenuator, arbitrary hot and cold temperatures of the load can be achieved. Another benefit with this approach is that the load remains the same independent of temperature. The drawback of the method is that the LNA needs to be connected at room temperature, which means a vacuum feedthrough and coaxial cable with a temperature gradient is needed between the cold attenuator and the LNA input, see Fig. 6.

Precise loss estimation of the vacuum feedthrough and cable is crucial for accurate noise measurement. The room temperature vacuum feedthrough can easily be measured with a VNA. The cable on the other hand is cold in one end and warm in the other. It is not fundamental to calibrate a VNA with one port inside a sealed vacuum chamber at cryogenic temperature. To determine the cable's loss, it was left open in the cold end, and measured with the VNA on the warm end. The S11 was then divided by two and smoothed to obtain the through loss of the cable. This method was tested on a warm cable and compared to a standard through measurement with very good agreement. The cable and feedthrough losses can be seen in Fig. 7.

To confirm the noise measurements, the LNAs were sent to an external laboratory for independent evaluation [7]. Two measurement methods were used. The first was based on two loads, where one was immersed in liquid nitrogen and the other at room temperature. The other was based on a standard N4002ASW noise source that was calibrated against the liquid nitrogen load. Both our and the Caltech measurement results can be seen in Fig. 8. The bias point of the first transistor was $V_{\mathrm{d}}=1.5 \mathrm{~V}, I_{\mathrm{d}}=45 \mathrm{~mA}$, and for stage $2+3$ it was $V_{\mathrm{d}}=1.8 \mathrm{~V}$, $I_{\mathrm{d}}=35 \mathrm{~mA}$, i.e. $17.5 \mathrm{~mA}$ per transistor.

The advantage of the liquid nitrogen setup is that the hot and cold temperatures are precisely known. The drawback is that the two loads might not present exactly the same impedance to the LNA. The advantage of the cold attenuator setup is that the load impedance doesn't differ between hot and cold state, but instead we have larger error in the noise power presented to the LNA. The error bars shown in Fig. 8 show the estimated absolute maximum error based on a total of $0.05 \mathrm{~dB}$ error in the measured losses of the vacuum feedthrough and cable in Fig. 7, and the estimation that the cryogenic to room temperature cable has an average temperature between 50 and $200 \mathrm{~K}$ (nominally $100 \mathrm{~K}$ ).

\section{CONCLUSIONS}

We have designed, fabricated and tested a room temperature LNA suitable for SKA band 1. The noise temperature and gain of the LNA was $10 \mathrm{~K}$ and $50 \mathrm{~dB}$, respectively. Noise temperature and S-parameters have the same shape and are close in magnitude to simulations. External measurements at an independent lab (Caltech) have confirmed the results.

\section{ACKNOWLEDGEMENT}

The authors would like to thank Dr. Sander Weinreb at Caltech for measuring the LNAs in his lab, and Dr. Bhushan Billade at Onsala Space Observatory, Chalmers University of Technology, for advice and measurement assistance. 
This research has been carried out in GigaHertz Centre in a joint project financed by Swedish Governmental Agency of Innovation Systems (VINNOVA), Chalmers University of Technology, and Low Noise Factory, Omnisys Instruments, Wasa Millimeter Wave and SP Technical Research Institute of Sweden.

\section{REFERENCES}

[1] The Square Kilometer Array SKA Home. Available: www.skatelescope.org

[2] M. W. Pospieszalski, "Extremely low-noise amplification with cryogenic FETs and HFETs: 1970-2004," IEEE Microw. Mag., vol. 6, pp. 62-75, Sep. 2005.

[3] J. Schleeh, G. Alestig, J. Halonen, A. Malmros, B. Nilsson, P. A. Nilsson, J. P. Starski, N. Wadefalk, H. Zirath, and J. Grahn, "Ultralow-power Cryogenic InP HEMT With Minimum Noise Temperature of $1 \mathrm{~K}$ at $6 \mathrm{GHz}, "$ IEEE Electron Device Letters, vol. 33, pp. 664-6, 2012.

[4] M. W. Pospieszalski, "Modeling of noise parameters of MESFETs and MODFETs and their frequency and temperature dependence," IEEE Trans. Microwave Theory Tech., vol. 37, pp. 1340-1350, 1989.

[5] J. Schleeh, H. Rodilla, N. Wadefalk, P. A. Nilsson, and J. Grahn, "Characterization and Modeling of Cryogenic UltraLow Noise InP HEMT," IEEE Transactions on Electron Devices, vol. 60, pp. 206-212, 2013.

[6] N4002A SNS Series Noise Source $10 \mathrm{MHz}$ to $26.5 \mathrm{GHz}$ (ENR $15 \mathrm{~dB})$. Available: http://www.keysight.com/en/pd$1000003572 \% 3$ Aepsg\%3 Apro-pn-N4002A/sns-series-noisesource-10-mhz-to-265-ghz-enr-15-db?cc=SE\&lc=eng

[7] "Dr. Sander Weinreb, California Institute of Technology (personal communication)." 